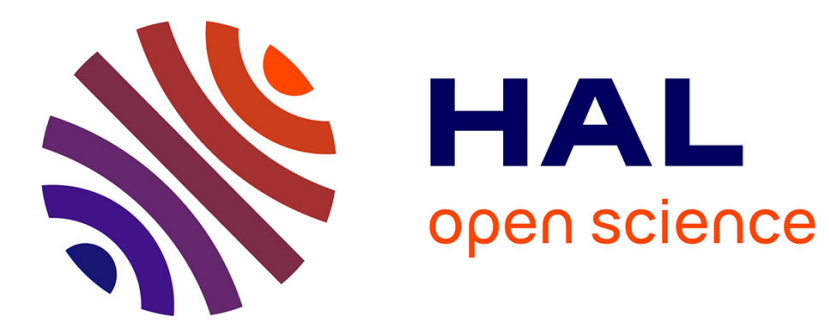

\title{
Naissance de la pédiatrie au 19 e siècle Jean-Jacques Baudon
}

\section{To cite this version:}

Jean-Jacques Baudon. Naissance de la pédiatrie au 19 e siècle. La Presse Médicale, 2017, 46 (4), pp.438 - 448. 10.1016/j.lpm.2017.01.021 . hal-01548972

\section{HAL Id: hal-01548972 \\ https://hal.sorbonne-universite.fr/hal-01548972}

Submitted on 28 Jun 2017

HAL is a multi-disciplinary open access archive for the deposit and dissemination of scientific research documents, whether they are published or not. The documents may come from teaching and research institutions in France or abroad, or from public or private research centers.
L'archive ouverte pluridisciplinaire HAL, est destinée au dépôt et à la diffusion de documents scientifiques de niveau recherche, publiés ou non, émanant des établissements d'enseignement et de recherche français ou étrangers, des laboratoires publics ou privés. 
Naissance d'une spécialité : la pédiatrie au $19^{\text {ème }}$ siècle

Naissance d'une spécialité : la pédiatrie au $19^{\text {ème }}$ siècle JJ Baudon

Faculté de Médecine Pierre et Marie Curie. Paris6

Département de Pédiatrie. Hôpital Armand Trousseau

26 Avenue du Dr Arnold Netter F75571 Paris Cedex 12

The advent of a newborn specialty: 19th Century Pediatrics

Baudonjj@aol.com

48940 caractères espaces compris.

Key words: history of medicine, history of pediatrics

JJ Baudon

Faculté de Médecine Pierre et Marie Curie. Paris6

Département de Pédiatrie. Hôpital Armand Trousseau

26 Avenue du Dr Arnold Netter F75571 Paris Cedex 12

The advent of a newborn specialty: 19th Century Pediatrics

Baudonjj@aol.com

48940 caractères espaces compris.

Key words: history of medicine, history of pediatrics 


\section{Résumé}

La pédiatrie a débuté dans des conditions sociales difficiles d'imaginer de nos jours. L'enfant au début du $19^{\text {ème }}$ siècle était considéré comme négligeable. Le taux de mortalité était très important, aggravé par le travail des enfants dès l'âge de 6 ans dans l'industrie textile. Dans les classes aisées, les petits étaient mis en nourrice loin de leurs parents, avec également un taux de décès dramatique. L'émergence de la pédiatrie s'inscrit dans le cadre des progrès des connaissances survenus durant le siècle : corrélation anatomo-cliniques, notion de contagion avant même la découverte des microbes puis naissance de la bactériologie. Pendant tout le siècle, les maladies infectieuses ont contribué grandement à la mortalité des enfants comme à celles des adultes par choléra, typhus, variole, diphtérie mais aussi rougeole, tuberculose. Les progrès enregistrés pendant la deuxième moitié du siècle ont résulté de l'apparition de I'hygiène, de l'antisepsie, de l'amélioration de la nutrition, de la prise en compte de l'enfant en tant que personne et de la nécessité de sa protection. En contraste, les thérapeutiques ont joué un rôle secondaire avec l'apparition de la sérothérapie et l'apparition des vaccins à l'aube du $20^{\mathrm{ème}}$ siècle. 


\begin{abstract}
Pediatrics began under the most unfavorable conditions that are difficult to imagine nowadays. Children at the start of the $19^{\text {th }}$ century were considered as negligible. The death rate was tremendous, increased by the work of children in factories as soon as 6 years of age in textile industries. In upper classes, infants were fed by a wet nurse, far from their parents and death rate was high as well. The emergence of pediatrics was the result of work carried out in adult medicine in the first half of the $19^{\text {th }}$ century: clinical anatomic method, knowledge of contagious diseases even before the discovery of bacteria, birth of bacteriology. During the whole century, infectious diseases contributed in a large part to children mortality, as that of adults, by cholera, typhus, variola, diphtheria, measles and tuberculosis. Progresses noted during the $2^{\text {nd }}$ part of the century resulted from beginning of hygiene, antisepsis, nutrition improvement, taking consideration of children as human being asking for protection. In contrast, therapeutics as serotherapy, vaccinations at the break of the 20th century played a secondary role.
\end{abstract}


Le $19^{\text {ème }}$ siècle marqué de profonds bouleversements politiques voit l'évolution des attitudes envers les enfants et les soins qui leurs sont donnés évoluer de façon progressive et lente sans les ruptures brusques observées dans l'histoire pendant le même temps. Cependant, en un siècle, l'espérance de vie s'allonge. Ce fait s'explique par les progrès économiques et sociaux survenus tardivement, l'apparition des notions d'hygiène et une plus grande considération pour les enfants à la fin du $19^{\text {ème }}$ siècle. En revanche, les premiers vaccins et médicaments découverts pendant cette période ont encore un impact limité sur la morbidité et mortalité des enfants même s'ils apportent à la médecine une certaine efficacité.

La pédiatrie en tant que branche spécialisée de la médecine n'a pas de réelle existence avant le milieu du $19^{\text {ème }}$ siècle ${ }^{[1]}$. Auparavant, elle est seulement une petite partie du courant principal de la médecine interne

\section{Place de l'enfant dans la société}

Le début de la pédiatrie s'inscrit dans des conditions sociales qu'il est difficile d'imaginer pour les enfants de nos jours. Pendant le $18^{\text {ème }}$ siècle, la mère, la nourrice et l'éducateur étaient les personnages importants mais pas l'enfant. Le nourrisson était considéré comme inéluctablement sujet aux maladies ${ }^{[2]}$. Au $19^{\text {ème }}$ siècle, les notions d'hygiène et l'émancipation des femmes commencent à émerger. Les relations entre pauvreté et maladies, évoquées par Franck à Padoue en 1790, sont affirmées par Villermé en France en 1840, Chadwich en Angleterre en 1842, Virchow en Allemagne en $1848^{[2]}$. La révolution industrielle, par les mouvements de population qu'elle entraîne, provoque l'abandon des zones rurales, l'entassement des populations dans les zones industrielles et des conditions de vie propices à la propagation des maladies ${ }^{[2]}$. De surcrôit, il existait un grand manque de 
sensibilité sur les conditions de vie des enfants ${ }^{[2]}$. Le concept de l'enfant malade demandant de soins particuliers et une attention spéciale a mis du temps à émerger.

Dans les classes pauvres, les enfants dans l'ensemble de l'Europe travaillaient dans l'industrie textile, la dentellerie en particulier dès 6 ans, et dans les mines dans des conditions épouvantables ${ }^{[3]}$. En 1833, en Angleterre, une loi créait un corps d'inspecteur du travail dans l'industrie textile afin de limiter le travail des enfants entre 9 et 13 ans à 9 heures par jour et en France la première loi sur le travail des enfants date de $1841^{[2]}$, à la suite des rapports de Villermé : «Sur la durée trop longue du travail des enfants dans beaucoup de manufactures » (lu à l'Institut de France 1837), suivi d'autres (1840) sur le travail des enfants dans les filatures de coton, les verreries. Peu d'attention était portée à ces rares règlements. La littérature montrait bien les conditions déplorables des enfants tout le long du $19^{\text {ème }}$ siècle. Les romans de Charles Dickens sont le reflet de ces conditions et du contraste entre enfants pauvres et enfants des classes aisées ${ }^{[2]}$. Dans les romans français, le travail des enfants dans les mines est illustré par Zola (Germinal 1885). Même dans les classes aisées, la première enfance était considérée comme négligeable. Dans la bourgeoisie et la noblesse, les enfants étaient placés en nourrice à la campagne dans des conditions d'hygiène et de confort qui n'avaient rien à voir avec celles de leur milieu d'origine. De nombreux parents apprirent ainsi la mort de leur enfant plusieurs mois après son décès .La plupart des nourrices étaient illettrées. Certaines n'étaient que des intermédiaires entre nourrices à la campagne et parents en ville. Il en résultait une énorme mortalité, surtout chez les enfants trouvés ${ }^{[4]}$. Cette habitude de mettre les enfants en nourrice a persisté une bonne partie du $19^{\text {ème }}$ siècle et n'a commencé à décliner qu'à partir de $1850^{[5]}$. Ainsi, madame Bovary (1857) place sa fille ; l'enfant d'Emma dormait à terre dans un berceau d'osier. « Il (Léon) lui semblait étrange de voir cette belle dame en robe de nankin tout au milieu de cette misère ». Dans le château des cours royales, l'enfant était alimenté par une nourrice. Les reines ignoraient naturellement 
l'effet de l'allaitement sur l'espacement des naissances. C'est ainsi que la reine Victoria eut 9 enfants entre 1840 et 1857 («You cannot really wish me to be the Mamma d'une nombreuse famille » sic, écrivait-elle à son oncle Léopold roi des Belges ${ }^{[6]}$. La pratique de l'enfant en nourrice persiste au début du $20^{\text {ème }}$ siècle. Dans le traité «Pratique Médico-Chirurgicale », Wallich en $1907^{[7]}$ consacre encore 3 pages aux conseils que les médecins doivent prodiguer aux mères qui confient leur enfant à une nourrice. Il rappelle les termes de la loi Roussel de 1874. Lorsque la nourrice vient se placer dans une famille (nourrice sur lieu), la loi prescrit que « toute personne qui vient se placer comme nourrice sur lieu est tenue de se munir d'un certificat du maire de sa résidence, indiquant si son dernier né est vivant et constatant qu'il est âgé de 7 mois révolus, ou, s'il n'a pas atteint cet âge, qu'il est allaité par une autre femme». L'enfant d'une nourrice abandonné par elle avant 7 mois, placé chez une nourrice au sein n'en succombe pas moins dans la proportion de un sur deux ${ }^{[7]}$.Si un médecin est appelé à donner ses conseils sur le choix d'une nourrice, il doit se préoccuper non seulement du nourrisson, mais aussi de la nourrice elle-même et de son enfant ${ }^{[7]}$. La syphilis était la grande crainte, considérée comme l'origine de bien des atteintes neurologiques : »il convient de ne pas oublier que les manifestations contagieuses de la syphilis héréditaire peuvent encore se montrer d'une façon assez tardive dans le premier ou le second mois après la naissance ». L'auteur insiste sur l'examen de la nourrice, non seulement sur l'état de ses seins mais aussi sur la recherche des signes cliniques de syphilis.

\section{Evolution des idées et progrès médicaux durant le $19^{\text {ème }}$ siècle}

Les progrès de la pédiatrie ont suivi l'évolution des idées générales sur les processus pathologiques. A la fin du $18^{\text {ème }}$ siècle, les premières maladies infantiles ont été isolées et décrites comme une entité bien que bon nombre fussent déjà connues depuis longtemps mais 
volontiers confondues entre elles ${ }^{[1]}$. La méthode anatomo-clinique a ouvert le développement explosif de la médecine au $19^{\text {ème }}$ siècle. Précédée par le travail de Morgagni (1761) qui le premier a développé l'idée de la localisation anatomique des processus morbides, Bichat (1771-1802) a émis le concept des tissus. Laënnec a fondé la séméiologie grâce à son invention du stéthoscope et la confrontation des données de l'examen clinique et des constatations anatomiques post mortem. En pédiatrie, les applications de cette méthode ont été développées par Billard (1800-1832) ${ }^{[1]}$ puis Barthez et Rillet dans leur traité de Médecine des Enfants (1843) ${ }^{[8,9]}$. Cependant, ils se rendirent compte que les corrélations anatomocliniques pouvaient être en défaut. L'autopsie pouvait ne révéler que des lésions terminales ${ }^{[1,4]}$.Autour des années 1850 , l'application du microscope à l'étude anatomique prit le relais et dégagea d'autres perspectives ${ }^{[4]}$. R Virchow (1821-1902) fournit les éléments d'une conception profondément rénovée de la pathologie. Son livre sur la pathologie cellulaire (1858) Die Cellular Pathologie établit le concept de Omnis cellule e cellula et devint l'une des bases scientifiques de la pédiatrie au même titre que celles d'autres disciplines.

La notion de contagion, avant la découverte des microbes par Pasteur, était controversée dans la médecine savante. Trois grandes théories ${ }^{[4]}$ étaient en présence depuis le $17^{\text {ème }}$ siècle: l'air vicié chargé de miasmes, le ressort ou tonus de l'organisme et celle du contagium vivum, mettant en cause des agents vivants invisibles. Cependant, dès le $18^{\text {ème }}$ siècle, à la suite de la grande épidémie de peste de Marseille (1720) puis d'autres, la notion de contagiosité va émerger et justifier des mesures d'isolement, de quarantaine, d'incinération des habits et couchage, en particulier ceux ayant des phtisiques, dans les pays de l'Europe du sud. La théorie des germes fut formulée à partir de 1840 par F Henlé (1809-1885) que les allemands considèrent comme le précurseur de Pasteur. Après la publication de la Théorie des Germes par Pasteur, une véritable révolution s’opéra dans la compréhension des maladies infectieuses. La pathologie bactérienne était née. Après la découverte par Pasteur du vibrion 
septique (1877), du staphylocoque (1880), du pneumocoque (1881), d'autres germes étaient mis en évidence. Entre 1881 et 1914, les agents de plus de 30 maladies infectieuses ont été isolés ${ }^{[2]}$, en particulier le bacille de la tuberculose par Koch (1882), le germe de la diphtérie par Klebs (1883) et Loeffler (1884), le bacille de la typhoïde par Eberth (1880) ${ }^{[4]}$, du choléra par Koch $(1884)^{[10]}$, de la peste par Yersin $(1894)^{[10]}$. Les premières notions sur le mode d'action des agents infectieux se développèrent. Le rôle fondamental des toxines était de plus en plus avancé pour la plupart des maladies infectieuses et tentait d'être distingué des autres facteurs d'agressivité .des germes comme l'invasion bactérienne. La première notion de virus, particule filtrable à l'inverse des bactéries, a été acquise en 1899 sur le virus de la mosaïque du tabac mais il faudra attendre le $20^{\text {ème }}$ siècle pour identifier des amas de particules virales dans les cellules cutanées de varioles animales en $1904-1906^{[10]}$ et les années 30 pour que fut établie l'origine virale des encéphalites, de la grippe et d'autres maladies ${ }^{[1]}$.

Les sciences physico-chimiques commencèrent à intéresser une minorité de médecins qui éloignèrent progressivement la médecine des théories philosophiques, vitalisme et autres ${ }^{[4]}$. Dutrochet (1776-1847) et Magendie (1783-1855) montrèrent que les lois régissant la perméabilité cellulaire étaient d'ordre physico-chimique. D'autres faits fondamentaux de la physiologie et de la chimie organique ont été établis par Ludwig (1816-1895) et Liebig (18031876). Ce dernier recruté aux USA, introduisit l'analyse chimique dans tous les domaines de la biologie. La physiologie prit son plein essor avec Claude Bernard qui développa le concept de milieu interne qui eut, mais plus tard, une influence majeure sur le traitement des enfants malades. Son livre Introduction à l'étude de la médecine expérimentale (1865) ouvrit le champ à la médecine expérimentale. La physiologie a fait un grand bond en avant avec les travaux de Heubner qui construisit le premier calorimètre respiratoire ${ }^{[1]}$ (1894) et établit les besoins énergétiques de l'enfant. Les explorations radiologiques en pédiatrie débutèrent rapidement après la découverte des rayons X par Roentgen en 1896 mais leur usage était 
encore très restreint malgré la création d'une première unité pour les enfants à Graz en 1897 [1]

La thérapeutique était encore limitée. La découverte de quelques médicaments, quinine, digitale, bismuth, morphiniques n'a sans doute pas joué un rôle majeur en pédiatrie. Les premiers vaccins contre la variole, la rage puis la typhoïde ont été découverts pendant cette période. La sérothérapie durant la dernière décennie du siècle a entraîné une réduction substantielle de la mortalité au cours de plusieurs maladies infectieuses. Les premiers antiseptiques sont apparus avant même l'ère pastorienne, en ordre dispersé et sujets à controverses et polémiques. La désinfection des instruments, des plaies et des surfaces opératoires a fait appel à l'alcool camphré, à l'acide phrénique en solution ou en vaporisations. A Guérin imagine le pansement ouaté pour empêcher l'infection par les germes aériens. Mais il reste des partisans du pansement malpropre qui croient que « l'asticot mange le vibrion ${ }^{[11]}$. Dans les sociétés savantes, on discute des mérites respectifs de l'iodoforme, du bichlorure de mercure, du permanganate de potassium et du chlore et du soufre pour désinfecter linges et literies ${ }^{[11]}$.

Les découvertes bactériologiques fondamentales sont venues étayer les notions d'hygiène, déjà défendues vigoureusement par Semmelweis à Vienne (1847).puis le chirurgien J Lister à Glasgow (1865) L’antisepsie était née.

\section{Morbidité et mortalité des enfants}

Au $18^{\text {ème }}$ siècle, la mortalité des enfants était extrêmement élevée. En Angleterre, Richard Watt a étudié la mortalité des enfants par maladie entre 1783 et 1812 et a conclu que plus de la moitié étaient décédés avant l'âge de 10 ans et parmi ceux-ci, plus du tiers étaient morts de variole, les autres de rougeole et autres infections ${ }^{[1]}$. La vaccination a contribué à faire baisser 
les décès liés à la variole mais les autres infections ont pris le relais. Chez les nourrissons, l'abandon de l'allaitement maternel a été la cause du décès de la plupart d'entre eux. Dans les asiles pour enfants, le taux de mortalité était effrayant de $50 \%$ à $99 \%{ }^{[5]}$. Au $19^{\text {ème }}$ siècle, l'industrialisation a été un facteur très important de morbidité et mortalité. Outre le travail précoce des plus grands, le travail des femmes a eu une influence déterminante sur la santé des nourrissons. A partir de la $2^{\text {ème }}$ moitié du $19^{\text {ème }}$ siècle, la mortalité infantile a augmenté de nouveau avec la révolution industrielle ${ }^{[9]}$. En maintenant les mères hors du foyer, ceux-ci étaient confiés à des mains mercenaires. Les femmes exposées aux conditions les plus insalubres transmettaient facilement leurs maladies aux plus petits. L'expansion des maladies vénériennes a pu aussi jouer un rôle. En 1860-61, le taux de mortalité infantile (avant 1 an) était de 141 pour 1000 naissances vivantes en Suède, 149 en Ecosse, 170 en Angleterre et 223 en France ${ }^{[1]}$. Les différences s'expliquent pour les pays du nord de l'Europe par les différences de taux d'industrialisation et de surcroît pour la France par l'abandon des enfants en nourrice ${ }^{[1,12]}$. Ces taux de mortalité infantile sont à comparer au taux actuel en France : 3,3 \%o en 2014 mais aussi dans le monde : Sierra Leone 117 \%, Angola ou en République Démocratique du Congo $100 \%{ }^{[12]}$. Heureusement, du $18^{\text {ème }}$ siècle au début du $20^{\text {ème }}$ siècle le taux de mortalité infantile a baissé régulièrement à partir du milieu du siècle : en France, il était de 167 pour mille en 1881, 139 pour mille en 1900, en Angleterre, les chiffres correspondants étaient de 129 pour mille et 138 pour mille puis 118 pour mille en 1907 ; aux USA, ce taux était de $160 \%$ en $1900^{[13]}$. Le taux de mortalité infanto-juvénile (c'est-à-dire avant 5 ans) a aussi baissé de façon spectaculaire. Ainsi à Londres $^{[1]}$, il était de $66 \%$ en $1730-79,37,7 \%$ en $1780-1829$ et $16,8 \%$ en $1911-15$. Cette baisse a été plus particulièrement marquée à partir de 1870 et surtout 1900. Ces progrès sont dus non seulement au développement de l'hygiène mais aussi à toutes les actions de santé publique en France, Angleterre et USA ${ }^{[13]}$. En 1884, F Marbeau créa la première crèche pour 
les mères qui travaillaient suivie de 9 autres. Le mouvement gagna l'Autriche, l'Italie, l'Allemagne. Ces initiatives, parallèlement aux lois de protection des enfants contribuèrent grandement à la baisse de la mortalité infantile. Parallèlement, dans la littérature naquit un grand mouvement d'intérêt pour l'enfant, dont les représentants les plus illustres ont été V Hugo, C Dickens.

Une expérience sociale remarquable illustre bien l'efficacité des mesures en faveur de l'enfance. A Villiers le Duc, un village de Côte d'Or, les maires Morel de Villiers père et fils firent chuter la mortalité infantile de 20\%-30\% en 1804-54 à 0 en 1893-1903 grâce à la déclaration des grossesses, leur surveillance et celle des enfants par des sages-femmes, la pesée des nourrissons, la surveillance de l'alimentation et des nourrices, la déclaration des maladies. Cette expérience eut une influence notable en Angleterre ${ }^{[1]}$

Les maladies infectieuses à la fin du $19^{\text {ème }}$ siècle demeurèrent une cause importante de mortalité touchant principalement les enfants. Des épidémies de choléra, typhus et variole sont survenues encore durant tout le $19^{\text {ème }}$ siècle ${ }^{[2,10]}$. La fréquence des épidémies de variole variait d'un pays à l'autre en fonction du taux de vaccination ${ }^{[2]}$. Après la guerre de 1870-71, une épidémie de variole en Allemagne toucha 175000 personnes, adultes et enfants et fit 100000 morts ${ }^{[2]}$. La scarlatine et la rougeole était des maladies sévères. A Vienne en 1855, la mortalité des malades admis à l'hôpital fut de $31 \%$ pour la rougeole et de $32 \%$ pour la scarlatine $^{[2]}$

Plusieurs lois au cours du $19^{\text {ème }}$ siècle firent obligation aux médecins de déclarer certaines maladies infectieuses. En 1822 (loi du 3/03/1822 et ordonnance du 7/08/1822) on exigeait des médecins, sous peine de sanctions sévères, l'obligation de déclarer toute maladie pestilentielle (peste, fièvre jaune, choléra) provenant de l'étranger ${ }^{[11]}$. En 1892, la loi (30/11/1892 et arrêté du 23/11/1893) imposait aux médecins d'informer les autorités de la présence de plusieurs 
maladies épidémiques, au nombre de 14 , tuberculose et syphilis exclues ${ }^{[11]}$. Parmi celles-ci, on notait la typhoïde, le typhus, la scarlatine, la rougeole, la diphtérie. Ces déclarations permettent d'avoir une idée plus précise du fardeau des maladies infectieuse. En Europe, Russie et pays balkaniques exceptés, au début du 20 ème siècle, la rougeole a tué 700167 personnes (254/an/million d'habitants), la scarlatine 470235 (171/an/million d'habitants), la coqueluche 661743 (240/an/million d'habitants), la diphtérie 589250 (214/an/million d'habitants) et la variole 90668 (33/an/million d'habitants) ${ }^{[4]}$ En raison des épidémies, un corps spécial de médecins fut créé, investi par l'administration de fonctions officielles et rétribués en tant que tels ${ }^{[11]}$. La diphtérie est le cas exemplaire des progrès décisifs durant cette période

\section{Les maladies infectieuses}

La diphtérie était une maladie redoutable qui tuait beaucoup d'enfants et d'adultes. Le bacille agissait par sécrétion d'une toxine et la fabrication de fausses membranes adhérentes envahissant les voies aériennes supérieures, en particulier le larynx-le croup-provoquant une asphyxie par obstruction. L'importance de la synthèse de toxine avec des fausses membranes extensives, des muqueuses œdématiées et hémorragiques, une fièvre élevée et un état général très altéré, distinguait les formes malignes. Les progrès décisifs sont survenus à cette époque dans la compréhension et la prise en charge de cette maladie. Dans l'ordre chronologique, Bretonneau (1826) montra l'unité des différentes formes cliniques et proposa la trachéotomie systématique dans les formes laryngées ${ }^{[9,15]}$. Après la découverte du bacille par Klebs et Löffler (1884), son mode d'action toxinique a été élucidé par Roux et Yersin (1888-90). Berhing a découvert la sérothérapie (1890) ${ }^{[15]}$ dont l'efficacité a été rapportée par Roux en 1894 ${ }^{[8]}$. Dans le même temps, la trachéotomie, pour lutter contre l'asphyxie, était supplantée par la mise au point du tubage laryngé (intubation) (Bouchut 1858, O’Dwyer 1885) ${ }^{[9,15]}$. 
Avant la sérothérapie, le taux de mortalité de la diphtérie était de l'ordre de $69 \%$ et après son introduction de $18 \%{ }^{[2]}$. Cependant, le nombre de malades restait identique et seule la vaccination par l'anatoxine de Ramon (1923-1925) a permis une chute majeure du nombre de cas (1525 en 1956, 46 en 1970) ${ }^{[4]}$ et sa disparition en France actuellement. Néanmoins, ces progrès n'ont pénétré que très lentement dans la France profonde. Plutôt que de tenter l'aventure de la trachéotomie, le médecin de campagne préférait assez souvent, aspirer les fausses membranes par un bouche-à- bouche extrêmement dangereux pour lui-même ${ }^{[11]}$ La variole était encore au $19^{\text {ème }}$ siècle une maladie redoutable, souvent mortelle. La maladie existait très probablement depuis le plus haute antiquité, en Inde notamment ${ }^{[10]}$. Transmise de siècle en siècle, au $18^{\text {ème }}$ siècle la maladie fut particulièrement grave en Europe avec une mortalité infantile élevée ${ }^{[10]}$. Elle représentait un véritable fléau dans les populations pauvres des grandes villes. Elle fut responsable de la perte de prés du quart de la population française ${ }^{[10]}$. En 1796, la Prusse compta plus de 26000 victimes de la variole et la Russie environ 2 millions ${ }^{[10]}$. Le déclin de la variole n'a commencé en Europe que dans la seconde moitié du $19^{\text {ème }}$ siècle ${ }^{[10]}$ avec encore une épidémie en $1870-71^{[16]}$. Pendant le siège de Paris, la variole entraîna le décès de 6500 personnes- le nombre d'enfants parmi celles-ci n'est pas précisé- et 1'hospitalisation de plus de 8000 militaires ${ }^{[4]}$. Par contre, dans l'armée allemande vaccinée, la mortalité ne dépassa pas 278 hommes ${ }^{[4]}$.La maladie s'éteignit progressivement en Europe et Amérique du Nord à partir du premier quart du $20^{\text {ème }}$ siècle ${ }^{[10]}$. La variole était une maladie terriblement contagieuse, de contamination exclusivement interhumaine, par contact direct ou par des tissus souillés, l’homme étant le seul réservoir du virus.

La maladie débutait par une fièvre élevée et des céphalées pendant 2 à 5 jours puis une atteinte du nez, de la gorge puis des lésions cutanées débutant au visage et gagnant en une marche descendante l'ensemble du corps ${ }^{[16,17]}$. Les lésions, simples taches érythémateuses au début évoluaient rapidement vers des pustules. Le nombre des pustules ou leur caractère 
hémorragique caractérisait les formes malignes. Ainsi dans la variole confluente la mortalité était de $60 \%$, dans les formes hémorragiques de prés de $100 \%$. Chez les femmes enceintes, la mortalité était de $60 \%$ et les enfants non vaccinés mourraient presque tous ${ }^{[16]}$. Dans les formes dites bénignes, la maladie guérissait en 3 semaines à 1 mois, en laissant des cicatrices indélébiles ${ }^{[16]}$. Le caractère viral de la variole n'a été démontré qu'en 1929 (Goodpasture), le virus identifié ultérieurement et son $\mathrm{ADN}$ entièrement séquencé. La variole a été déclarée éradiquée en 1977 grâce à la vaccination ${ }^{[10,17]}$.

La rougeole, maladie de l'enfant, préférentiellement entre 2 et 5 ans, était quasi obligatoire et l'est restée jusqu'à la généralisation récente de la vaccination. Séparée de la scarlatine qu'à la fin du $18^{\text {ème }}$ siècle, son étude et ses complications ont été complétées dans la seconde moitié du $19^{\text {ème }}$ siècle ${ }^{[18]}$. Les complications respiratoires de la rougeole, laryngite, pneumonie, faisait toute sa gravité et étaient d'autant plus à redouter que l'enfant était plus jeune. Le risque de ces complications chiffré au début du $20^{\text {ème }}$ siècle était estimé à $35 \%$ avant 1 an, $26 \%$ dans les 2 premières années, $20 \%$ de 2 à 5 ans ${ }^{[18]}$. Les encéphalites étaient beaucoup plus rares. La rougeole était considérée comme une maladie grave : la mortalité au début du $20^{\text {ème }}$ siècle était de $1,8 \%$ dans les formes simples, beaucoup plus élevée chez les nourrissons et les enfants avec un terrain débilité ${ }^{[18]}$. Une idée de sa gravité au $19^{\text {ème }}$ siècle peut être donnée par les taux de mortalité actuels dans les pays les plus pauvres qui peuvent atteindre $25 \%$ voire $50 \%$ chez les nourrissons. La rubéole n'a été considérée comme une maladie spécifique que dans la seconde moitié du $19^{\text {ème }}$ siècle, volontiers confondue avec d'autres éruptions de l'enfance, roséole infantile et autres éruptions virales ${ }^{[4]}$.

La tuberculose présente dès la préhistoire a été identifiée à partir du $18^{\text {ème }}$ siècle : un quart des décès de l'adulte était dus à cette maladie ${ }^{[19]}$ Cependant, à partir de la révolution industrielle, lorsque la démographie s'est rapidement développée, la tuberculose fut encore plus un problème majeur de santé publique ${ }^{[19]}$. Laennec le premier établit l'unicité des 
différentes localisations de la tuberculose ${ }^{[4]}$ et décrivit sa lésion élémentaire, le granulome, travaux confirmés par Rilliet et Barthez ${ }^{[9]}$. Villemin montra le caractère infectieux et inoculable de la maladie, avant la découverte du bacille par Koch ${ }^{[20]}$. La découverte des réactions cutanées à la tuberculine, étape capitale dans la détection de la tuberculose infantile date du début du $20^{\text {ème }}$ siècle ${ }^{[4]}$. La compréhension de sa physiopathologie explique pourquoi l'enfant est particulièrement exposé. La primo-infection tuberculeuse fait suite à l'inhalation de bacilles surtout lors d'un effort de toux. Le sujet contaminant est d'autant plus contagieux qu'il est porteur de lésions cavitaires pulmonaires et qu'il est dans un lieu confiné ${ }^{[19]}$. L'enfance est habituellement le moment du premier contact de l'hôte avec le BK ${ }^{[21]}$. La lésion primaire ou nodule, située dans le parenchyme pulmonaire va le plus souvent disparaitre (tuberculose latente), plus rarement elle augmente de taille, s'étend dans le parenchyme et/ou dans la plèvre (pleurésie) ${ }^{[19]}$. A partir du foyer pulmonaire initial, la tuberculose peut gagner les ganglions thoraciques ou par voie sanguine se disséminer dans les articulations, la méninge. Le développement de la réponse immunitaire spécifique empêche le plus souvent cette évolution : 10\% des patients après la primo-infection développeront une tuberculose maladie ${ }^{[19]}$. La notion de tuberculose latente et la possibilité de tuberculose maladie, se développant ultérieurement était déjà bien établie au début du $20^{\text {ème }}$ siècle ${ }^{[20]}$. L'enfant qui contractait une primo-infection avant 5 ans était (et demeure) particulièrement exposé aux formes disséminées les plus sévères, miliaire et méningite. Les tuberculoses ostéoarticulaires et viscérales étaient souvent plus tardives ${ }^{[22]}$. Dans les formes aigues généralisées, les miliaires et les méningites, la mort était constante. La scrofule associait des adénites froides du cou et des lésions cutanées en regard, décrites encore dans les traités du début du $20^{\text {ème }}$ siècle ${ }^{[20]}$ qui pouvaient s'observer dés la première enfance, surtout entre 5 et 15 ans ${ }^{[20]}$. Les lésions cutanées étaient dites scrofules et les tuméfactions ganglionnaires les écrouelles. 
Dans les descriptions qui en étaient faites, il est difficile de distinguer ce qui revenait au BK, à des lésions de surinfection à pyogènes ou à des adénites infectieuses non tuberculeuses. Les tuberculoses ostéoarticulaires les plus fréquentes étaient la coxalgie et le mal de Pott ou tuberculose vertébrale ${ }^{[23]}$. L'évolution de ces tuberculoses, fréquentes chez l'enfant, se faisait vers l'ankylose et la déformation articulaire souvent après des années d'immobilisation. Le décès n’était pas rare par généralisation tuberculeuse.

La fièvre typhoïde mérite encore une place parmi les nombreuses maladies infectieuses courantes au $19^{\text {ème }}$ siècle et au-delà. En l'absence de traitement, la typhoïde était souvent mortelle. Bretonneau le premier entre 1819 et 1829 a montré la constance et la spécificité des lésions intestinales et isolé les manifestations cliniques au sein des autres fièvres ${ }^{[4,9]}$. Les formes de l'enfant ont été décrites à partir de 1839 (Rilliet). La découverte du bacille par Eberth dans les selles puis des bacilles paratyphiques A et B en 1900 ne permettait pas de diagnostiquer tous les cas avant l'hémoculture et le sérodiagnostic de Widal (1896).

Le premier vaccin a été le vaccin antivariolique. Les premiers essais de protection contre la variole, la variolisation, consistaient dans l'injection volontaire d'une forme bénigne de variole, destinée à protéger le sujet contre l'atteinte de la variole grave ${ }^{[4,10]}$. Cette pratique introduite en Angleterre durant le $18^{\text {ème }}$ siècle puis en France à partir de 1750, comportait un risque de dissémination était estimé de 2\% à 5\%. Jenner en 1796 a publié les résultats obtenus par la vaccination. Sa découverte reposait sur l'observation que les vachers ayant contracté la vaccine ou cow pox étaient protégés contre la variole ${ }^{[10]}$. La vaccination se faisait de bras à bras. Elle fut introduite en Europe continentale à peu prés en même temps au début du 19 ème siècle. Cependant, elle se répandit très lentement en raison des difficultés et des réticences à sa mise en œuvre. En 1897, la mise au point du vaccin sec puis de la lyophilisation contribuèrent à sa généralisation ${ }^{[10]}$ qui amena le déclin de la variole ; la vaccination des 
enfants devint obligatoire en France en 1902. Le vaccination contre la rage, mis au point par Pasteur (1885), marque une étape importante dans l'histoire des vaccins. Après avoir obtenu une atténuation du virus rabique par passages successifs à l'animal, Pasteur l'injecta à un enfant mordu par un chien enragé afin de le préserver de la maladie compte tenu de la longue durée d'incubation. Cependant, la rage était un exemple particulier du fait même du temps que prend le virus pour gagner l'encéphale et déterminer une encéphalite. Cette expérience fut d'ailleurs très contestée dans l'immédiat et par la suite ${ }^{[24]}$. Le vaccin contre la typhoïde a été mis au point par Wright (1896) et diffusé en France en $1910^{[4]}$. Son impact a été considérable pendant la $1^{\text {ère }}$ guerre dans l'armée française. En pédiatrie, en revanche le vaccin compte tenu de ses effets secondaires n'a pratiquement pas été employé.

La pathologie interne a eu également un grand développement pendant tout le $19^{\text {ème }}$ siècle. Le siècle a été marqué par la description des maladies ou syndromes neurologiques qui portent encore le nom de leurs auteurs : West $(1871)^{[1,2]}$, Duchenne (1858), Bourneville (1880) ${ }^{[8]}$. Little (1862) a décrit les conséquences des accouchements traumatiques et de 1'asphyxie périnatale ${ }^{[25]}$. En rhumatologie, le rhumatisme articulaire aigu, ses complications cardiaques et les nodules ont été distingués des arthrites chroniques (Still 1897) ${ }^{[26]}$. En cardiologie, malgré le stéthoscope, les descriptions demeuraient essentiellement anatomiques post-mortem ; ainsi Bouillaud (1840) décrivit les lésions inflammatoires du péricarde et des valves ${ }^{[28]}$ puis Cheadle l'ensemble des lésions (1889) ${ }^{[27]}$. Les premières descriptions de malformations cardiaques datent également du $19^{\text {ème }}$ siècle : tétralogie de Fallot $\left(1885^{\text {[8], }}\right.$ communication interventriculaire (Roger $1889^{{ }^{[8]}}$. En gastroentérologie, la première description de la maladie cœliaque (Gee) est de $1888^{[4]}$ 
Les problèmes nutritionnels ont joué un grand rôle dans le développement de la pédiatrie.

L'allaitement maternel et les alimentations artificielles ont suscité beaucoup de discussions.

Dans la deuxième moitié du $18^{\text {ème }}$ siècle, le suédois Rosenstein soutint, comme Rousseau, que le nourrisson devait être alimenté par le lait de sa propre mère ${ }^{[1]}$. En 1907, les recommandations étaient les mêmes ${ }^{[7]}$. A la fin du $18^{\text {ème }}$ siècle, certains aliments pouvaient être préférés aux laits animaux pour nourrir les jeunes enfants ${ }^{[1,5]}$. Ainsi, des mélanges de différentes farines de plusieurs céréales, plus ou moins sucrées, enrichis en viandes diverses voire bière ou vin étaient préconisés. Ils étaient administrés grâce à des ustensiles en faïence spécialement conçus. Cette alimentation entraînait des carences majeures, jouant un rôle important dans la mortalité de ces enfants. A partir du milieu du $19^{\text {ème }}$ siècle, le développement de la calorimétrie a permis de mesurer l'azote et le CO2 éliminés au cours du métabolisme des protéines, des lipides et des glucides et ainsi la ration calorique de l'enfants sain et hypotrophique (Heubner 1885) ${ }^{[4]}$. Au début du $20^{\text {ème }}$ siècle, l'hygiène de l'alimentation avait grandement progressé. Les conceptions sur l'allaitement artificiel avaient quelque peu changé à la suite d'analyses montrant des compositions très différentes du lait de femme et du lait de vache. Cependant, malgré les modifications apportées au lait de vache en le coupant avec de l'eau et en le sucrant, sa composition était encore très loin des laits infantiles modernes. La malnutrition, aggravée par les conditions socio-économiques, était très courante. Les carences vitaminiques, en premier lieu en vitamine D étaient fréquentes. Le rachitisme a été décrit au $17^{\text {ème }}$ siècle (Glisson 1650). Les déformations des membres qu'il induit ne pouvaient passer inaperçues. La révolution industrielle a amené une explosion de l'incidence du rachitisme ${ }^{[28]}$. L'absence d'ensoleillement dans des logements insalubres, la pollution et le confinement des enfants expliquaient sans doute cette véritable épidémie qui était telle que peu d'enfants échappaient au rachitisme. Sans être mortelle, la maladie prédisposait aux pneumonies et aux convulsions par carence en calcium. La cause du 
rachitisme a été débattue pendant tout le $19^{\text {ème }}$ siècle. Les lésions histologiques osseuses et cartilagineuses ont été décrites par Virchow (1853) puis Schmorl (1909). Cependant, la découverte du facteur anti-rachitique, la vitamine $\mathrm{D}$, et de sa liaison avec l'ensoleillement fut très longue à émerger malgré les observations de Palm, médecin missionnaire au Japon, qui constata l'absence de rachitisme liée à l'ensoleillement, malgré la pauvreté des populations, et d'autre part la constatation de signes de rachitisme chez les lions du zoo de Londres à l'abri du soleil ! ${ }^{[28]}$. En Allemagne, en 1916, l’héliothérapie fut instituée. L'action anti-rachitique de 1'huile de foie de morue fut longtemps attribuée à la vitamine $\mathrm{A}^{[28]}$. Finalement, il fallut attendre les années 20 pour que fut découvert dans la peau, le 7 déhydrocholestérol, dont l'irradiation pas les UV détermine la synthèse de vitamine D. Le scorbut (carence en vitamine C) a été décrit chez l'enfant par Barlow en 1883 et attribué à la consommation de laits commerciaux bouillis ou condensés ${ }^{[5]}$. En revanche, ce n'est que dans la première décennie du $20^{\text {ème }}$ siècle que le béribéri (déficit en thiamine), la pellagre (déficit en niacine) et les conséquences oculaires de la carence en vitamine A ont été reconnus comme d'origine nutritionnelle. Funk en 1912, a établi la théorie des vitamines, substances normalement présentes dans l'alimentation en petites quantités, dont la carence détermine une maladie spécifique ${ }^{[29]}$ Les carences en oligoéléments étaient présentes dans certaines régions. Les Alpes, Pyrénées, Auvergne et Vosges, étaient touchées par une endémie »goitre-crétineuses ». En effet, 5 à 15 p 100 de la population, au milieu du siècle, présentaient des goitres et les familles comptaient une proportion anormale de crétins. Les médecins incriminaient les vents froids, les habitations humides et les mariages consanguins ${ }^{[11]}$. On sait maintenant que ces goitres avec hypothyroïdie étaient liés à une carence en iode. La supplémentation des eaux de boisson les a fait disparaître.

Les carences en fer étaient méconnues. Jusqu'en 1850, beaucoup de littérature et de fantaisies médicales ont couru sur la chlorose. Certains auteurs attribuaient le teint des jeunes 
adolescentes, jugé de couleur verte, à la virginité. D'autres faisaient valoir la distribution égale dans les deux sexes d'une anémie et insistaient sur une sorte d'anorexie nerveuse des adolescentes ${ }^{[30]}$. Le traitement empirique par le sulfate de fer a été institué en 1832, sous forme des pilules de Baud. A partir de 1850, la découverte de l'hémoglobine (Funk 1851) puis des méthodes colorimétriques d'étude du sang (Gowers 1878, Haldane 1901), ont permis de démembrer le cadre des anémies, sans pour autant faire taire rapidement les polémiques sur la chlorose ${ }^{[30]}$. Ce n'est qu'en 1911 qu'apparut la notion d'anémie par carence en fer, souvent liée à une alimentation du nourrisson uniquement à base de lait de vache et dans l'entre-deux guerres que fut démontrée la régénération du taux d'hémoglobine par le fer ${ }^{[30]}$

Avant l'anesthésie, la chirurgie des enfants était limitée aux domaines dans lesquels s'était déjà exercé le savoir-faire des chirurgiens des siècles précédents ${ }^{[4]}$. Des progrès techniques avaient été faits mais l'éventail des interventions ne s'était guère élargi et restait limité à la réduction et l'immobilisation des fractures et des luxations, le traitement empirique des brûlures et des plaies. Des appareillages de plus en plus élaborés étaient mis au point pour les scolioses. Ainsi était née une spécialité l'orthopédie. La chirurgie abdominale ne connaissait que quelques interventions simples et rapides, imposées par l'urgence ou quand tout espoir de survie était exclu ${ }^{[4]}$.

La découverte des premiers anesthésiques, protoxyde d'azote, vapeur d'éther et chloroforme a eu lieu dans la première moitié du $19^{\text {ème }}$ siècle. L'anesthésie a été appliquée aux enfants, même à de jeunes nourrissons au milieu du siècle ${ }^{[31]}$. La rapidité des effets des anesthésiants sur les enfants a été soulignée ${ }^{[31]}$.La mise au point de masques a grandement facilité leur utilisation chez l'adulte puis chez l'enfant. Leur utilisation a débuté au milieu du siècle par Guersant aux Enfants Malades et Delabarre aux Enfants Assistés. La mise au point du masque d'Ombredanne (1908), basé sur la ré-inhalation de l'air expiré enrichi en éther, a permis une 
meilleure application. Cependant, les anesthésiques comportaient une toxicité notable, hépatique pour le chloroforme, refroidissement des voies respiratoires pour l'éther. De surcroît, l'anesthésie était administrée par les étudiants, le garçon de salle, rarement par une infirmière. Il existait de nombreux accidents, syncope bleue ou blanche et les premiers, en particulier avec le chloroforme, ont entrainé des débats. Le critère du bon chirurgien au début du $20^{\text {ème }}$ était la rapidité de l'opération.

L'intervention de l'appendicite, grevée d'une lourde mortalité, a fait l'objet de longs débats pour l'adulte puis pour l'enfant. En raison du caractère le plus souvent catastrophique de l'appendicite non opérée, l'opération a fini par s'imposer en particulier sous l'influence de Dieulafoy de 1895 à $1906^{[4]}$. Beaucoup de techniques chirurgicales n'ont en fait été inventées qu'au début qu'au début du $20^{\text {ème }}$ siècle comme la pylorotomie sous-muqueuse pour les nourrissons atteints de sténose du pylore (Fredet 1906).

\section{Les définitions des maladies héréditaires et des maladies congénitales n'étaient pas}

\section{acquises.}

A l'heure actuelle, l'hérédité répond à une définition précise, la transmission par les parents à leurs enfants de caractères, inscrits dans les gènes, ou de qualités exprimées ou non ${ }^{[32]}$. Une maladie congénitale est une maladie avec laquelle l'enfant est né, qu'elle soit héréditaire, ou acquise pendant la vie intra-utérine et non transmissible comme embryopathie ou fœtopathie

${ }^{\text {[32] }}$. Une maladie familiale est une affection qui frappe plusieurs membres d'une même famille mais dont le caractère génétique n'est pas certain. Au $19^{\text {ème }}$ siècle, l'hérédité est une notion confuse. « Non seulement les particularités innées sont transmises héréditairement mais les particularités acquises le sont aussi » (Littré médical) ${ }^{[33]}$. C'est ainsi qu'il est fait un grand 
usage de la notion de syphilis héréditaire pour expliquer beaucoup de pathologies alors que la syphilis congénitale est une fœtopathie.

La plupart des maladies héréditaires étaient encore ignorées. La descendance de la reine Victoria a cependant illustré tôt l'arbre généalogique de l'hémophilie. Son fils Léopold a souffert très jeune d'hémorragies récurrentes qui ont été décrites dans les journaux médicaux en $1868^{[34]}$. Puis la reine transmit par l'intermédiaire de ses filles à ses descendants mâles l'hémophilie, maladie héréditaire liée à l'X, qui gagna la plupart des cours européennes. Sa petite fille, la tsarine, née de Hesse, transmit la maladie au tsarévitch.

La puériculture mit longtemps à émerger. Les soins aux nourrissons et la médicalisation des enfants cristallisèrent la défiance réciproque des femmes et des médecins ${ }^{[4]}$. Au cours du $19^{\text {ème }}$ siècle, les médecins intervinrent dans le domaine réservé de la puériculture ${ }^{[4]}$. Les critiques concernaient l'emmaillotage rigoureux par le maillot complet qui condamnait l'enfant à l'immobilité absolue et comprimait excessivement les membres et le tronc. Cette immobilisation favorisait les luxations de hanche. Les médecins firent adopter le maillot à l'anglaise qui laissait aux bébés une plus grande liberté. Dans le domaine de l'alimentation, le sevrage brutal fut remplacé par une alimentation plus progressive qui réduisit la surmortalité infantile liée aux gastro-entérites. Au début du siècle, l'idée était répandue qu'il ne fallait pas trop laver les enfants car la crasse protège. Après 1871, certains médecins demandèrent que l'on épouille les nourrissons de leur vermine et qu'on nettoie soigneusement les biberons et tétines ${ }^{[4]}$. Les nouvelles institutrices enseignaient aux fillettes les rudiments de puériculture. La protection de l'enfance a émergé dans la deuxième moitié du siècle. Plusieurs lois furent votées sous l'impulsion de députés médecins. T Roussel et A Morvan sont à l'origine de la loi du 23 novembre 1874 sur la protection des enfants en nourrice qui tarda à être appliquée ${ }^{[4]}$. Paul Bert fit introduire l'enseignement de l'hygiène et la pratique de l'éducation physique 
dans les écoles publiques. Ces lois furent suivies par celles sur les enfants maltraités ou abandonnés (24/07/89), sur le travail des jeunes dans l'industrie (2/11/1892), sur l'assistance médicale gratuite (15/07/93). Parallèlement, à partir de la Suisse, des lois sur le repos des femmes enceintes, 15 jours avant le terme et 6 semaines après, furent promulguées dans toute l'Europe. Les premières consultations de nourrissons ont ouvert à Paris en 1892, organisées par P Budin puis la distribution de lait (œuvres de la goutte de lait).

La maltraitance des enfants, enfants battus et abus sexuels, ont été largement décrits par Tardieu (1860) ${ }^{[3]}$

Si la pédiatrie mit longtemps à apparaitre, la période néonatale resta négligée plus longtemps encore $^{14]}$. Le nouveau-né passait directement des mains de la sage-femme dans celles de la nourrice. La pathologie néonatale était intégrée à l'obstétrique dans le prolongement du suivi de la grossesse et de l'accouchement ${ }^{[35]}$. Il fallut attendre 1828 pour que paraisse le premier ouvrage réellement consacré au nouveau-né, le « Traité des maladies des enfants nouveau-nés et à la mamelle » de Billard ${ }^{[4]}$ puis celui de Valleix. La précision des analyses de Billard et le recours à la vérification anatomique sont les témoins d'une rigueur déjà toute scientifique ${ }^{[4]}$. Il fut le créateur de la puériculture. En 1901, Ballantyne distinguait déjà la pathologie embryonnaire, la fotale, les soins pré et post natals ${ }^{[4]}$. Cependant, ce n'est qu'après deuxième guerre que la néonatologie va émerger comme une sous spécialité.

Au milieu du $18^{\text {ème }}$ siècle, sont parus les premiers travaux sur le poids et la taille des nouveaunés (Roederer). Progressivement, à partir de 1815, se répandit l'usage de peser les nouveaunés ${ }^{[35]}$. A partir des données sur les enfants bien portants nés à terme, on commença de distinguer les «faiblesses de naissance », sans faire la part de la prématurité et du retard de la croissance intra-utérine. L'importance et le danger du refroidissement ont été reconnus à partir du milieu du $19^{\text {ème }}$ siècle. Denucé fit construire en 1852 des berceaux à double paroi 
pour y faire circuler de l'eau chaude ${ }^{[4,36]}$. Cependant, le véritable créateur des couveuses fut Tarnier $^{[4,35,36]}$ qui avait vu des incubateurs à poulets au jardin d'acclimatation. Les premiers modèles ont été réalisés en 1880-81 et utilisé à la Maternité de Port Royal puis améliorés par Mme Henry, sage-femme et par Pinard et Budin. Parallèlement, Tarnier et surtout Budin insistèrent sur l'importance de l'allaitement maternel ; Budin mit au point la méthode du gavage $^{[35]}$.

Les accidents mécaniques de la naissance, ce qu'il est convenu d'appeler le traumatisme obstétrical, étaient connus depuis longtemps. Pendant le $19^{\text {ème }}$ siècle, la description des différentes lésions traumatiques devint plus précise : bosse séro-sanguine et céphalhématome ${ }^{\text {[35] }}$ (Valleix 1838), paralysie obstétricale du plexus brachial, paralysie faciale.

Les détresses respiratoires du nouveau-né, avec leurs divers mécanismes, «forme blanche », correspondant à l'absence première de mouvements respiratoires et « formes bleues » avec cyanose et asphyxie ont bien été étudiées au $19^{\text {ème }}$ siècle ainsi que les lésions pulmonaires et cérébrales correspondantes ${ }^{[4,35]}$. Les premières mesures thérapeutiques ont été proposées : Billard conseille de désobstruer le pharynx avec le doigt ; De Salle souligne l'utilité de la respiration bouche à bouche. L'insufflation par différents instruments est née progressivement dans le courant du siècle. En fait, dans leur conception, ces mesures sont restées d'actualité jusque dans les années 1950. Les conséquences lointaines des lésions cérébrales liées à l'asphyxie ont été établies pour la première fois par Little à Londres en 1862. L'infection néonatale au début du siècle était encore très mal connue. Elle était intimement liée au milieu du $19^{\text {ème }}$ siècle à l'infection puerpérale. La découverte de l'antisepsie à la suite des travaux de Lister, Semmelweiss, Budin a permis une régression spectaculaire des fièvres puerpérales ainsi que des omphalites. Le tétanos néonatal, la syphilis congénitale ont été décrits dans la seconde moitié du $19^{\text {ème }}$ siècle. 
L'ictère physiologique était connu dès la fin du $18^{\text {ème }}$ siècle ${ }^{[35]}$ mais sa cause demeurait inconnue. L'ictère hémolytique par incompatibilité fœto-maternelle était ignoré et il faudra attendre 1903 (Schmorl) pour la description de l'ictère nucléaire.

\section{Emergence de la pédiatrie en tant que spécialité, son enseignement, les premiers}

\section{hôpitaux d'enfants}

Les premiers livres consacrés à la pédiatrie sont apparus à la fin du $18^{\text {ème }}$ siècle : Rosen von Rosenstein en suédois (1764), Underwood en anglais (1784), Girtanner en allemand (1794) puis dans la première moitié du $19^{\text {ème }}$ siècle, Billard (Traité des maladies des enfants nouveaunés et à la mamelle 1828), Barthez et Rilliet (Traité clinique et pratique des maladies des enfants 1843) ${ }^{[2]}$. Dans la seconde moitié du siècle, les livres de pédiatrie augmentèrent en nombre et épaisseur, comme celui de Gerhardt : Handbuch der Kinderkrankheiten 1887-89 en 6 volumes avec des contributions internationales qui en font une somme des connaissances sur la pédiatrie de l'époque ${ }^{[2] .}$

Les premiers hôpitaux pédiatriques ont été fondés au $19^{\text {ème }}$ siècle. En 1850, 25 existaient déjà, le plus ancien était l'hôpital des Enfants Malades de Paris (1802), suivi du Pavillon pédiatrique de la Charité de Berlin (1830) et ceux de Saint Petersbourg (1834), Vienne et Breslau $(1837)^{[2,8]}$. De 1850 à 1879, 67 hôpitaux pédiatriques ou départements pédiatriques d'hôpitaux généraux ouvrirent en Europe ${ }^{[2]}$, dont le premier britannique créé par West : «Hospital for Sick Children » (1852) et plusieurs aux USA : Philadelphie (1855), Boston 1869) ${ }^{[14]}$. Ce développement témoigne de l'intérêt croissant porté à la médecine des enfants. Elle eut cependant pour inconvénient la grande fréquence des infections nosocomiales. L'enseignement de la pédiatrie à la fin du $19^{\text {ème }}$ siècle a été marqué par la création des premières chaires universitaires ${ }^{[2,4]}$. A Paris, la première « Chaire de Clinique de Maladies des Enfants » fut crée en 1879 avec Parrot pour premier titulaire. A Vienne, le premier 
«Professor Ordinarius » fut Widerhofer (1884). A la même époque, Henoch à Berlin créa l'enseignement de la pédiatrie, suivi par Heubner, titulaire de la $1^{\text {ère }}$ chaire de pédiatrie (1894). Durant ces deux dernières décennies du $19^{\text {ème }}$ siècle, il en fut de même dans les autres pays européens, en Italie à Padoue (1882), Naples (1887), Rome (1897), en Espagne à Madrid (1895). En Grande Bretagne et aux USA, le système des chaires n'existait pas mais l'enseignement de la pédiatrie commença à la même époque avec Barlow University College (1895-1907), Still à Londres et Smith (1861) à New York ${ }^{[7,13,25]}$

La création de sociétés savantes consacrées à la pédiatrie commença à la fin du $19^{\text {ème }}$ et au début du $20^{\text {ème }}$ siècle, en Allemagne, en Angleterre « Society for the Study of Diseases of Children » (Londres 1900) ${ }^{[8]}$, aux USA «American Pediatric Society » $(1888)^{[13]}$ et en France. Des journaux exclusivement consacrés à la pédiatrie ont commencé leur parution au cours du $19^{\text {ème }}$ siècle ${ }^{[1]}$ et les premiers congrès au début du $20^{\text {ème }}$.

\section{Conclusion}

De la fin du $18^{\text {ème }}$ siècle jusqu'à la première guerre mondiale, des progrès majeurs ont été accomplis dans le domaine de la médecine des enfants. Ces progrès ont eu lieu malgré des périodes de stagnation voire de régression. Ils tiennent au développement souvent simultané de plusieurs facteurs volontiers intriqués, qui sont loin d'être tous d'ordre purement médical comme l'hygiène des enfants, l'asepsie, l'isolement des contagieux, la prise de conscience de l'enfant comme être humain, la connaissance des besoins nutritionnels, la description de carences et l'importance de l'allaitement maternel, la découverte de beaucoup d'agents infectieux microbiens et la mise au point des premiers vaccins, l'émergence de la pédiatrie comme spécialité autonome. 
Les conditions de la médecine des enfants avant la première guerre mondiale sont encore présentes dans les pays les plus pauvres de la planète. Les vaccins, les toxines microbiennes, la prévention sont plus que jamais d'actualité. 


\section{Références}

1 Garrison FH. History of Pediatrics in: Abt-Garrison History of Pediatrics. Saunders édit Philadelphia 1965 chap. I p 1-172

2 Ballabriga A. One century of Pediatrics in Europe in "History of Pediatrics 1850-1950" edited by BL Nichols, A Ballabriga, N Kretchmer. Nestlé Nutrition Workshop Series. Vevey/Raven Press New York 1991, vol 22, p 1-21

3 Labbé J. Ambroise Tardieu: the man and his work on child maltreatment a century before Kempe. Child Abuse Negl 2005;29:311-324

4 Huard P, Laplane R Histoire Illustrée de la Pédiatrie 3 tomes. Editions Roger Dacosta- Paris 1981

5 Fomon SJ. Infant feeding in "History of Pediatrics 1850-1950" edited by BL Nichols, A Ballabriga, N Kretchmer. Nestlé Nutrition Workshop Series. Vevey/Raven Press New York 1991, vol 22, p 77-89

6 Editorial. If Queen Victoria had known about LAM. Lancet 1991;337:703-4

7 Wallich V Allaitement in « Pratique Médico-chirurgicale » Brissaud E, Pinard A, Reclus P. Masson édit Paris 1907, T1

8 Laplane R. French Pediatrics in One century of Pediatrics in Europe in "History of Pediatrics 1850-1950” edited by BL Nichols, A Ballabriga, N Kretchmer. Nestlé Nutrition Workshop Series. Vevey/Raven Press New York 1991, vol 22, p 39-48

9 Shulman ST. The history of pediatric infectious diseases. Pediatr Res 2003;55:163-176.

10 Dedet JP. Les épidémies. De la peste noire à la grippe A/H1N1. Dunod édit 2010

11 Léonard J La vie quotidienne du médecin de province au XIXème siècle. Hachette 1977 12 WWW.ined.fr La mortalité infantile en France 
13 Stoll BJ. American Pediatric Society 2013 presidential address: $125^{\text {th }}$ anniversary of the American Pediatric Society-lessons from the past to guide the future. Pediatr Res 2013; $74: 466-472$

14 Pearson HA. Pediatrics in the United States in "History of Pediatrics 1850-1950" edited by BL Nichols, A Ballabriga, N Kretchmer. Nestlé Nutrition Workshop Series. Vevey/Raven Press New York 1991, vol 22, p 55-63

15 Marfan AB. Leçons cliniques sur la diphtérie. Masson édit Paris 1905

16 Tollemer L. Variole in «Pratique Médico-chirurgicale » Brissaud E, Pinard A, Reclus P. Masson édit Paris 1907, T VI

17 Red Book 2012. Smallpox (Variola). Report of the Commitee on Infectious Diseases. American Academy of Pediatrics edit Elk Village 29th edition, 647-650

18 Tollemer L. Rougeole in « Pratique Médico-chirurgicale ». Brissaud E, Pinard A, Reclus P. Masson édit Paris 1907 T V

19 Leroy H, Revest M, Tattevin P. Histoire naturelle de la tuberculose. Rev Prat 2012;62: $479-86$

20 Lereboullet P. Tuberculose in : «Pratique Médico-chirurgicale » «Pratique Médicochirurgicale » Brissaud E, Pinard A, Reclus P. Masson édit Paris 1907 T VI

21 Delacourt C. Particularités de la tuberculose chez l'enfant. Rev Prat 2012;62:504-6

22 Gerbeaux J. Tuberculose primaire de l'enfant. Flammarion édit. Paris 1967

23 Lapointe A. Coxalgie in : «Pratique Médico-chirurgicale » Brissaud E, Pinard A, Reclus P. Masson édit Paris 1907, T II

24 Ligon BL Louis Pasteur : a controversial figure in a debate on scientific ethics. Semin Pediatr Infect Dis 2002;13:134-141

25 Millichap JJ, Millichap JG. Child neurology: past, present, and future. Part I: history. Neurology 2009; 73:e31-e33 
26 Schaller JG. The history of pediatric rheumatology. Pediatr Res 2005; 58:997-1007

27 Noonan JA. A history of pediatric specialties: the development of pediatric cardiology.

Pediatr Res 2004;56:298-306

28 Harrison HE. Rickets in "History of Pediatrics 1850-1950" edited by BL Nichols, A

Ballabriga, N Kretchmer. Nestlé Nutrition Workshop Series. Vevey/Raven Press New York 1991, vol 22, p 159-170

29 Barness LA. Vitamin deficiencies in "History of Pediatrics 1850-1950" op cit p 171-179

30 Woodruff CW. Anemias in «History of Pediatrics 1850-1950” op cit p 181-187

31 Sabourdin N. Histoire de l'anesthésie pédiatrique : des origines jusqu'à la fin du XIX ${ }^{\mathrm{e}}$

siècle. Ann Franç Anesth Réanim 2013;32:e237-e242

32 Garnier, Delamare. Dictionnaire des termes de médecine. Maloine édit Paris, $26^{\text {ème }}$ édition, 2000

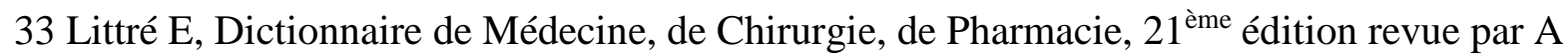
Gilbert. JB Baillière édit 1908.

34 Rushton AR Leopold the «bleeder prince » and public knowledge about hemophilia in Victorian Britain. J Hist Med Allied Sci 2012;67:457-490

35 Salle BL, Vert P. Néonatologie : passé et présent. Bull Acad. Natle Méd 2013;197:12311242

36 Dunn PM. Stéphane Tarnier (1828-1897), the architect of perinatology in France. Arch Dis Child Fetal Neonatal. 2002;86:F137-F 139. 


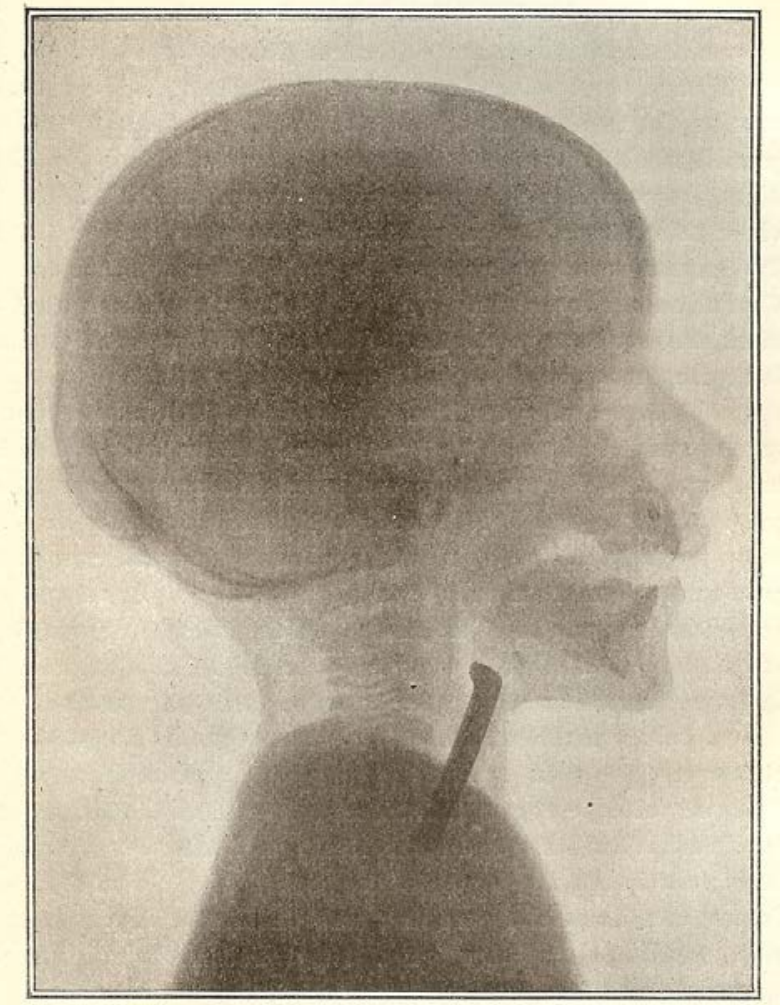

Figure 1 : tubage laryngé. In Marfan. Leçon cliniques sur la diphtérie. Masson édit. Paris 1905. 


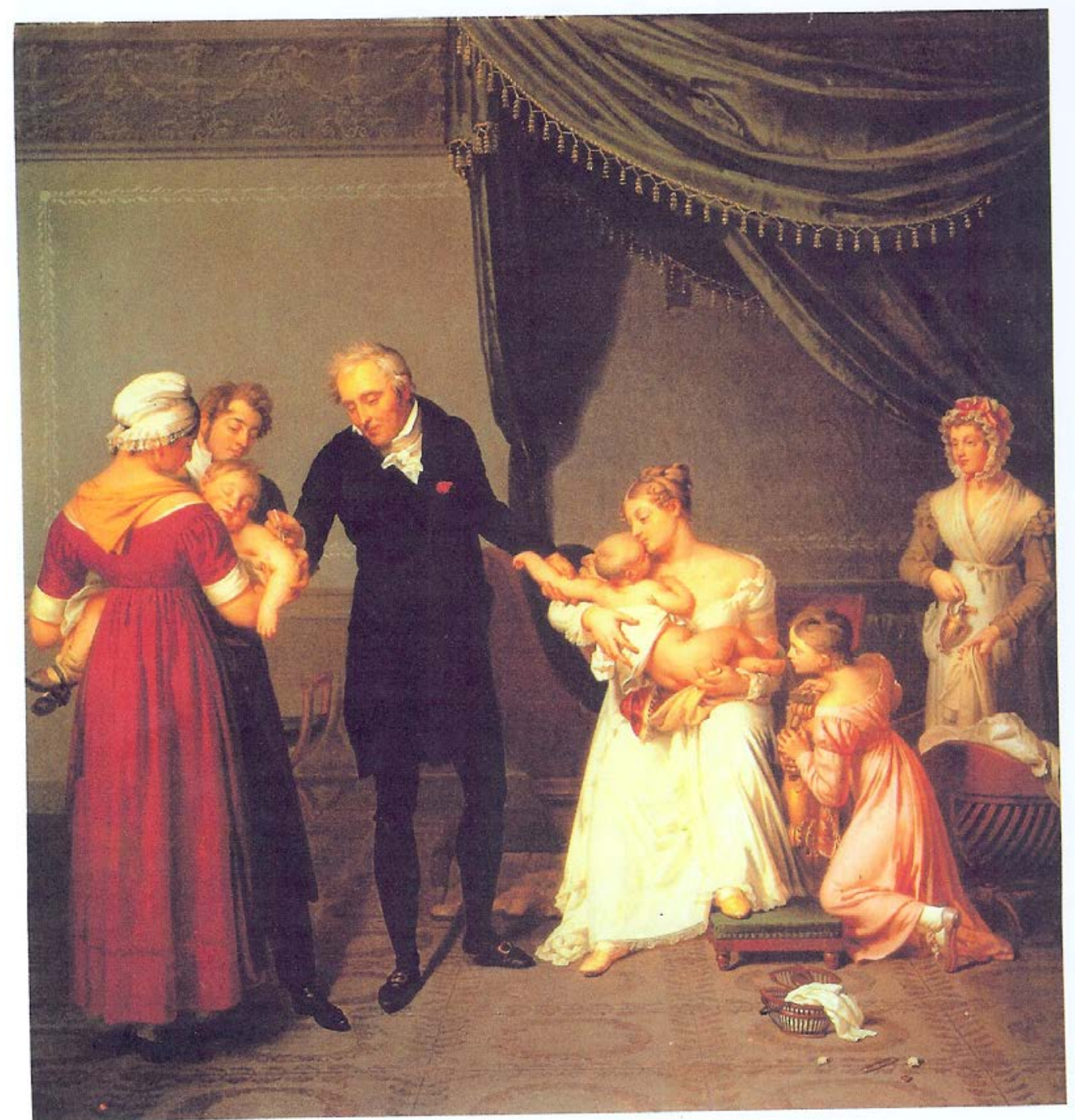

Figure 2 : La vaccine au château de Liancourt. Desbordes, Musée de l’Assistance Publique 


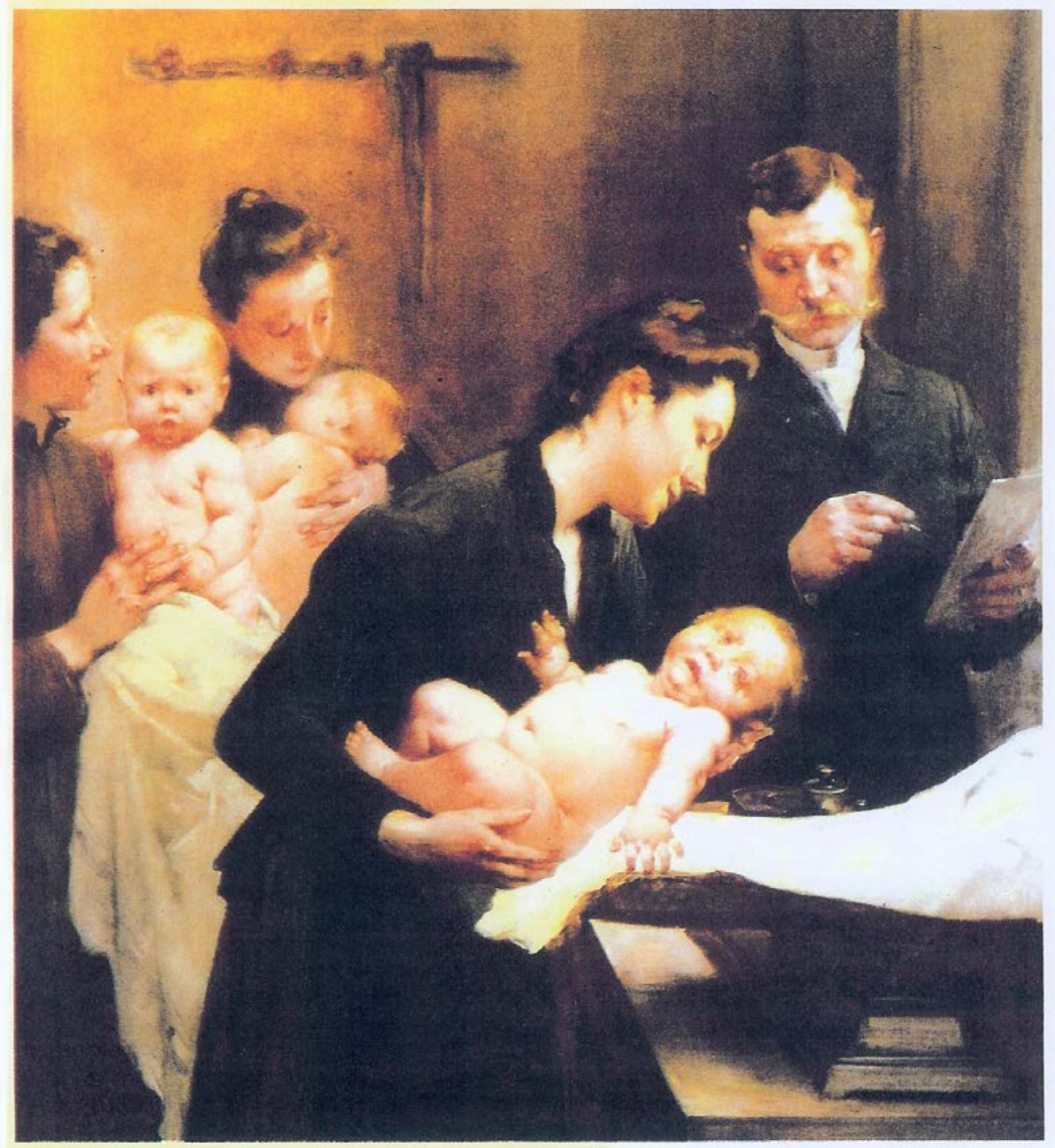

Figure 3 : La goutte de lait de Belleville du Dr Variot : la pesée - Geoffroy, musée de l'Assistance Publique 